\title{
Design And Preparation of New Decellularized Extracellular Matrix/Alginate Composite Membranes For Biomimetic Bioinks Via Supercritical Carbon Dioxide Fluid Treatments
}

Ching-Cheng Huang ( $\nabla$ junas.tw@yahoo.com.tw)

Ming Chuan University

\section{Research Article}

Keywords: Supercritical Carbon Dioxide, microstructure, decellularization, alginate, composite membranes

Posted Date: June 29th, 2021

DOl: https://doi.org/10.21203/rs.3.rs-622568/v1

License: (c) (i) This work is licensed under a Creative Commons Attribution 4.0 International License. Read Full License 


\section{Abstract}

Tissue-engineering was important and popular which combine medical applications and engineering materials knowledge, just like decellularization techniques were employed to remove the cellular components from porcine elastic cartilages, leaving a native decellularized extracellular matrix(dECM) composition and architecture integrity of largely insoluble collagen, elastin, and tightly bound glycosaminoglycans. Particularly, an extraction process of supercritical carbon dioxide $\left(\mathrm{ScCO}_{2}\right)$ was used to remove cellular components from porcine skins. The porcine skins must remove lipids and other impurities by using $\mathrm{ScCO}_{2}$ procedure. In this study, a series of new composite membranes with decellularized scaffolds could be designed and obtained from porcine skin tissue by using supercritical carbon dioxide fluid technology. The retain decellularized extracellular matrix (dECM) and integrity scaffold-structure could be observed in the new composite membranes. This work provides a simple and time-saving method process for preparation of biomedical composite membranes with dECM scaffolds for biomimetic bioinks, which were further characterized by Fourier transform infrared spectroscopy (FTIR), thermo-gravimetric analysis (TGA), and scanning electron microscope(SEM).

\section{Introduction}

Tree-dimensional (3D) bioprinting shows potential in tissue engineering and regenerative applications due to its overwhelming advantages over other approaches. In order to promote the functions of bioprinted tissues, the development of novel and versatile bioinks will have crucial implications[1]. Natural derived materials are famous for the excellent biocompatibility and abundance, among which sodium alginate mixed with gelatin has been widely used as bioink for extrusion-based 3D bioprinting[2]. Despite advance in bio-printing and bio-fabrication during the past decade, fabricating complex and functional tissue constructs that mimic their natural counterparts still remains a challenge[3]. Bioink optimization is considered as one of main challenges in cell-laden 3D bioprinting.

Numerous materials have been proposed, modified and empolyed for medical bioprinting applications such as scaffolds for skin and bone tissue reconstruction such as synthetic materials and natural materials[4-16]. Sodium alginate is a naturally occurring biopolymer extracted from different species of marine brown algae[12]. Sodium alginate, the marine-derived polysaccharide, can offer many advantages over synthetic polymers since they interact under relatively mild temperature and $\mathrm{pH}[13]$. Alginate shows a wide range of biomedical applications especially in cell immobilization and tissue regeneration because of its outstanding properties such as biodegradability and biocompatibility[14]. Alginate has attractive features including ease of gelation with divalent cations such as calcium ions for enhanced stability of $\mathrm{Ca}$-alginate[15].

Decellularized extracellular matrix (dECM) scaffolds had a lot of collagens, which constitute the main structural element of the $\mathrm{dECM}$, provide tensile strength, regulate cell adhesion, support migration, and direct tissue development. Dense connective tissue is an abundant source of dECM scaffolds, which can be prepared and purified by a defatting and decellularizing procedure[17-20]. The objectives of the 
present manuscript are to provide a new design of composite materials containing alginate and decellularized extracellular matrix(dECM) scaffolds with high stability and their expected effects on the biological properties of the scaffolds in orthopedics and gene therapy[17-20].

In this study, the treatments combined with supercritical carbon dioxide and specific enzymes to prepare dECM scaffolds. Furthermore, a series of new composite materials containing dECM scaffolds and alginate were designed and prepared. The composite membranes containing collagen scaffolds for biomimetic bioinks must be characterized by Fourier transform infrared spectroscopy(FTIR), thermogravimetric analysis (TGA), and scanning electron microscope(SEM) to get the results of identifications, thermal stabilities, and microstructures.

\section{Experimental}

\subsection{Materials}

The chemicals used in the work, such as enzymes of papain (Sigma-Aldrich Company), Sodium alginate(Sigma-Aldrich Company), Calcium chloride (Fluka), TritonX-100(Shanchai-Lingfeng Chemical Company), $\mathrm{NaOH}($ Sigma-Aldrich Company), acetic acid, and alcohol.

\subsection{Treatments with supercritical carbon dioxide before preparation of a decellularized extracellular matrix}

Supercritical fluids of carbon dioxide $\left(\mathrm{ScCO}_{2}\right)$ was empolyed for preparation of new designed decellularized extracellular matrix scaffolds in this study. The $\mathrm{ScCO}_{2}$ was employed before enzyme treatments for removing most fatty acids and tissues[4].

\subsection{Preparation of a decellularized extracellular matrix}

The steadily thickness of about $0.5 \mathrm{~mm}$ of tissue sample could be obtained from porcine skin by using a designed tissue-cutting machine(Taiwan PARSD Pharm. Tech. Consulting Ltd Co. and Kuin Biotech. Ltd Co.). Samples are soaked in $25 \% \mathrm{NaOH}(\mathrm{aq})$ for $2 \mathrm{hr}$ with magnet mixer, followed by $0.5 \mathrm{U} / \mathrm{ml}$ papain(aq) at $25^{\circ} \mathrm{C}$ for $2 \mathrm{hr}$. The resulting samples were washed with doubledistilled water under ultrasonic wave to remove residual fat and organic matter. The resulting sample was frozen for $6 \mathrm{hrs}$ and then lyophilized (EYELA, FD-5N) overnight with the use of a freeze dryer at $0.1-0.2$ torr at a freeze-drying temperature of $-45^{\circ} \mathrm{C}$. A designed collagen scaffold, dECM, could be obtained.

\subsection{Preparation of decellularized extracellular matrix/alginate composite membranes}

In this study, a series of composite membranes with dECM scaffolds were prepared based on the various weight ratios of alginate and dECM (alginate/ dECM: 100/0, 95/5, 90/10, 85/15, 80/20). Briefly, the desired amount of dECM powder was first dispersed completely in $40 \mathrm{~mL}$ of doubledistilled water with the homogenizer at $26,000 \mathrm{rpm}$ for $3 \mathrm{~min}$. Then, alginate aqueous solution was homogenized thoroughly with the dispersed dECM solution at 26,000 rpm for $3 \mathrm{~min}$. The alginate/dECM solutions were then molded and frozen for 6 hrs and then lyophilized (EYELA, FD-5N) overnight. A series of new 
alginate/dECM composite membranes were obtained such as ALG/dECM1N, ALG/dECM2N, ALG/dECM3N, and ALG/dECM4N (Table 1).

\subsection{Preparation of cross-linked decellularized extracellular matrix/alginate composite membranes}

The decellularized extracellular matrix/alginate composite membrane is soaked in $\mathrm{CaCl}_{2}$ aqueous solution with various concentrations for different crosslinking reactions with magnet mixer. The crosslinked decellularized extracellular matrix/alginate composite membranes were then molded, frozen, and dried by the same procedure described above. A series of designed decellularized extracellular matrix/alginate composite membranes could be obtained(Table 1).

\section{Results And Discussion}

\subsection{Fourier Transform Infrared Spectroscopy Analysis of alginate/dECM composite memebranes}

From the FTIR analysis of the original porcine skin[Figure 1(A)], absorptions bands at 1452, 1400, 1337, 1240,1203 , and $1080 \mathrm{~cm}^{-1}$ were attributed to the amides III containing $\delta\left(\mathrm{CH}_{2}\right), \delta\left(\mathrm{CH}_{3}\right), v(\mathrm{C}-\mathrm{N})$, and $\delta(\mathrm{N}-$ $\mathrm{H})$ absorptions of collagens in the original porcine skin. Amides I and amides II absorptions were found at 1632 and $1551 \mathrm{~cm}^{-1}$, respectively. The absorption band at $3301 \mathrm{~cm}^{-1} \delta(\mathrm{C}-\mathrm{H})$ was attributed to the fatty acid of the original porcine skin. The absorption band at $1744 \mathrm{~cm}^{-1} \delta(C=0)$ was attributed to the fatty acid. The absorption bands of fatty acids could not be observed in Fig. 1(B), demonstrating the effectiveness of the supercritical carbon dioxide treatment.

Table1. Preparation of new ALG/dECM composite memebranes with collagen scaffold 


\begin{tabular}{|c|c|c|}
\hline Sample No. & ALG/dECM (w/w) & {$\left[\mathrm{CaCl}_{2}\right]$} \\
\hline ALG/dECMONa) & $100 / 0$ & - \\
\hline ALG/dECM 1N ${ }^{a)}$ & $95 / 5$ & - \\
\hline ALG/dECM2N $\mathrm{N}^{\mathrm{a})}$ & $90 / 10$ & - \\
\hline ALG/dECM3Na) & $85 / 15$ & - \\
\hline ALG/dECM4Na) & $80 / 20$ & - \\
\hline ALG/dECMOL $\left.{ }^{\mathrm{a}, \mathrm{b}}\right)$ & $100 / 0$ & $1 w t \%$ \\
\hline ALG/dECM1L $\left.{ }^{a, b}\right)$ & $95 / 5$ & $1 w t \%$ \\
\hline ALG/dECM2L $\left.{ }^{a, b}\right)$ & $90 / 10$ & $1 w t \%$ \\
\hline ALG/dECM3L ${ }^{\mathrm{a}, \mathrm{b})}$ & $85 / 15$ & $1 w t \%$ \\
\hline ALG/dECM4L $\left.{ }^{a, b}\right)$ & $80 / 20$ & $1 w t \%$ \\
\hline $\mathrm{ALG} / \mathrm{dECMOH}{ }^{\mathrm{a}, \mathrm{c})}$ & $100 / 0$ & $5 w t \%$ \\
\hline $\mathrm{ALG} / \mathrm{dECM} 1 \mathrm{H}^{\mathrm{a}, \mathrm{c})}$ & $95 / 5$ & $5 w t \%$ \\
\hline $\mathrm{ALG} / \mathrm{dECM} 2 \mathrm{H}^{\mathrm{a}, \mathrm{c})}$ & $90 / 10$ & $5 w t \%$ \\
\hline $\mathrm{ALG} / \mathrm{dECM} 3 \mathrm{H}^{\mathrm{a}, \mathrm{c})}$ & $85 / 15$ & $5 w t \%$ \\
\hline ALG/dECM4H $\left.{ }^{a, c}\right)$ & $80 / 20$ & $5 w t \%$ \\
\hline
\end{tabular}

a) ALG: alginate and dECM: Porcine Skins via $\mathrm{ScCO}_{2}$ and Papain Treatments.

b) Relative low concentration of $\mathrm{CaCl}_{2}(\mathrm{aq})$.

c) Relative high concentration of $\mathrm{CaCl}_{2}(\mathrm{aq})$.

Figure 1(C) showed the typical absorption bands of sodium alginate, mainly the $\mathrm{O}-\mathrm{H}$ stretching at 3,424 $\mathrm{cm}^{-1}$, pyranoid ring(sixmembered ring) $\mathrm{C}-\mathrm{H}$ stretching at 2,903 and 2,932 $\mathrm{cm}^{-1}, \mathrm{COO}$ symmetric stretching at $1595 \mathrm{~cm}^{-1}, \mathrm{COO}$ asymmetric stretching at $1,408 \mathrm{~cm}^{-1}, \mathrm{C}-0$ stretching at 1,338 and $1,298 \mathrm{~cm}^{-1}$, and C$0-C$ stretching at $1,094 \mathrm{~cm}^{-1}$. Figure 1 (D) still showed a remarkably absorption band at $2903 \mathrm{~cm}^{-1}$ in the spectra of ALG/dECM membranes which indicates that formation of the egg-box model due to the crosslinking with calcium ion has not occurred. If the formation of the egg-box model has occurred, the stretching vibration of $\mathrm{C}-\mathrm{H}$ in the six-membered ring of the calcium alginate molecule is limited and the corresponding absorption band cannot be observed in the spectrum. In the sodium alginate molecule, the stretching vibration absorption band of - $\mathrm{COO}$ - and $\mathrm{C}-\mathrm{O}$ is very weak. However, in the calcium alginate 
molecule, the -C-O-O-Ca-O-CO- structure makes the $\mathrm{C}-\mathrm{O}$ stretching vibration absorption increase and has an obvious absorption band at $1024 \mathrm{~cm}^{-1}$ as shown in Figure 1(C) and 1(D) which indicated that the formation of -CO-O-Ca-O-CO- structure in ALG/dECM membranes.

The FTIR spectroscopy analysis was carried out to confirm the incorporation of dECM in ALG/dECM composite membrane. In the spectrum of the ALG/dECM composite membrane, besides retaining the above mentioned bands of pure sodium alginate(ALG/dECMON). The characteristic bands of $\mathrm{ALG} / \mathrm{dECMON}$ at around $1595 \mathrm{~cm}^{-1}$ (the carbonyl (C=0) bond) and $1408 \mathrm{~cm}^{-1}$ (asymmetric and symmetric stretching peaks of carboxylate salt groups) were visible. It showed a stronger absorption band at 1595 $\mathrm{cm}^{-1}$ and two remarkably shoulders at $1632 \mathrm{~cm}^{-1}$ and $1537 \mathrm{~cm}^{-1}$, which were characteristic absorption of carbonyl groups of amide of dECM molecules, which confirmed the formation of ALG/dECM composite membrane effectively. For ALG/dECM composite membrane, the main absorption bands at around 1632 $\mathrm{cm}^{-1}$ (amide I, C-O, and C-N stretching), $1537 \mathrm{~cm}^{-1}$ (amide II) and $1242 \mathrm{~cm}^{-1}$ (amide III) were also observed. A higher absorption from $3600 \mathrm{~cm}^{-1}$ to $3200 \mathrm{~cm}^{-1}$ appeared in the spectrum of ALG/dECM scaffold, which suggested an increase of hydrogen bonds resulting from the interaction between dECM molecule and alginate(ALG) molecule. The results of FTIR indicated the presence of dECM in the hybrid scaffold as wells as the interaction between them.

\subsection{The Microstructure of Resulting Alginate/dECM Composite Memebranes with Collagen Scaffolds}

The microstructures of resulting membranes with dECM scaffolds were characterized by scanning electron microscope (SEM). Scanning electron micrographs of an original porcine skin and a dECM sample after treatment with supercritical carbon dioxide were showed in Fig. 2(A) and Fig. 2(B), respectively. The micro-scaffold structure could be observed in the dECM sample derived from porcine skin [Figure 2(B)]. The averaged diameter of microscafford was found in a range of $8 \sim 25 \mu \mathrm{m}$ [Figure 2(B)]. The different micro-scaffold shape with relative narrow boundaries was observed in scanning electron micrographs of ALG sample as shown in Fig. 2(C). The averaged diameter of narrow boundaries was found in a range of $1 \sim 3 \mu \mathrm{m}$. Furthermore, scanning electron micrographs of resulting new decellularized composite membranes were showed in Fig. 3(A) 3(D). The remarkable micro-scaffold structures were still observed in the composite membranes with various introduction ratios of $\mathrm{dECM}$ and ALG. With the increasing introduction ratio of $\mathrm{dECM}$ and $A L G$, the new combined micro-scaffold shapes of composite membranes were similar to the micro-scaffold shape of dECM. The new combined microscaffold shapes were observed with the smooth mixed boundary as shown in Fig. 3(D). The averaged diameter of smooth mixed boundary was found in a range of $1 \sim 35 \mu \mathrm{m}$ [Figure 3(D)]. The new combined micro-scaffold shapes could be proposed in the schematic diagrams as shown in Fig. 4 and Fig. 5.

\subsection{Thermal Stability of Resulting Alginate/dECM Composite Memebranes with Collagen Scaffolds}

Thermal stability of resulting scaffolds could be characterized by TGA. Thermogravimetric analysis of the alginate material maximum pyrolysis temperature $\left(T_{d M a x}\right)$ is lower than 300 degrees. In order to enhance the thermal stability of the designed composite membranes, the decellularized extracellular matrix (dECM) scaffolds were introduced. The maximum pyrolysis temperature $\left(T_{d M a x}\right)$ of the resulting 
composite membranes is higher than 300 degrees. The resulting composite membranes with dECM molecules would be new heat-resistant biomaterials as shown in Fig. 6 \& Table 1 .

TGA analysis of the dECM molecule was shown in Fig. 4. The main loss is presented in three different temperature ranges given by I: $\left(\left\langle 150^{\circ} \mathrm{C}\right)\right.$, II: $\left(150-200^{\circ} \mathrm{C}\right)$ and III:(200-450 $\left.0^{\circ} \mathrm{C}\right)$. The curve in I corresponded to the loss of the physisorbed and chemical water in the resulting dECM molecule with decellularized extracellular matrix scaffold, which represented the $25 \mathrm{wt} \%$ of the sample, which occurred at $40^{\circ} \mathrm{C}$. The following main loss, occurring in the range of temperatures II and III in the thermogram results, for the dECM molecule was observed at $180^{\circ} \mathrm{C}$ and $330^{\circ} \mathrm{C}$, respectively. The losses would be related to the combustion of $\mathrm{dECM}$ molecule.

TGA analysis of ALG/dECM membranes are shown in Fig. 5. The main loss is presented in two different

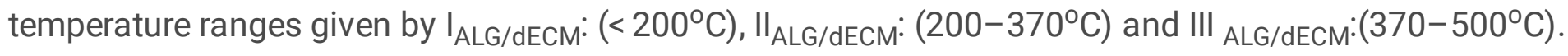
Initial weight loss up to $200{ }^{\circ} \mathrm{C}$ is found to be 15, 20, 20 and $5 \sim 13 \%$ for NaALG(ALG/dECMON), CaALG $(\mathrm{ALG} / \mathrm{dECMOH}), \mathrm{dECM}$, and ALG/dECM membrane respectively, due to the elimination of absorbed and bounded water molecules in the membrane. In case of ALG/dECM, weight loss after cross linking increases from 10 to $12 \%(\mathrm{dECM} / \mathrm{ALG} 4 \mathrm{~L}$ and dECM/ALG4H) as compared to the non-crosslinked sample(dECM/ALG4N). Similar observation is made for ALG before and after crosslinking (14-20\%). This increase may be due to more adsorption of water molecules present along with $\mathrm{Ca}^{2+}$ molecules while crosslinking with $\mathrm{CaCl}_{2}$ aqueous solution. However more interestingly, we observe that for $\mathrm{dECM}$ loaded ALG/dECM membrane samples, this increase in weight loss after crosslinking was less (3\%) as compared to ALG membrane(6\%). Further second stage $\left(\mathrm{II}_{\mathrm{ALG} / \mathrm{dECM}}\right)$ of weight loss as observed from 200 to $370^{\circ} \mathrm{C}$ corresponds to thermal degradation of ECM molecules due to the breakage of protein chain. The relative high $\mathrm{T}_{\mathrm{dmax}}(\mathrm{b})$ values of $\mathrm{ALG} / \mathrm{dECM}$ were observed at $350^{\circ} \mathrm{C}$ in second stage $\left(\mathrm{II}_{\mathrm{ALG} / \mathrm{dECM}}\right)$ comparing with the $330^{\circ} \mathrm{C}$ of $\mathrm{dECM}$ molecule. Also, the relative high $\mathrm{T}_{\mathrm{dmax}}(\mathrm{a})$ values of $\mathrm{ALG} / \mathrm{dECM}$ were observed at 270 $\sim 300^{\circ} \mathrm{C}$ in second stage $\left(\|_{\mathrm{ALG} / \mathrm{dECM}}\right)$ comparing with the $250^{\circ} \mathrm{C}$ of non-crosslinked ALG molecule. When the high concentration of $\mathrm{CaCl}_{2}(5 \mathrm{wt} \%)$ added, the relative high $\mathrm{T}_{\text {dmax }}(\mathrm{a})$ value of crosslinked ALG was observed at ca. $270{ }^{\circ} \mathrm{C}$ comparing with the $250{ }^{\circ} \mathrm{C}$ of non-crosslinked ALG molecule and $252^{\circ} \mathrm{C}$ of slight crosslinked ALG molecule (1wt\%) as shown in Fig. 4(A) and 4(B). Furthermore, when the high concentration of $\mathrm{CaCl}_{2}(5 \mathrm{wt} \%)$ added, the relative high $\mathrm{T}_{\mathrm{dmax}}(\mathrm{a})$ values of high crosslinked $\mathrm{ALG} / \mathrm{dECM}$ composite membranes were observed at ca. $300^{\circ} \mathrm{C}$ comparing with the $270^{\circ} \mathrm{C}$ of high crosslinked ALG molecules(5wt\%) as shown in Figs. 7 and 5(C). It would be due to the association between the ALG molecule and dECM molecule. Similarly, the relative high $\mathrm{T}_{\mathrm{dmax}}(\mathrm{b})$ value of crosslinked ALG/dECM would be observed at ca. $370^{\circ} \mathrm{C}$. Particularly, another much higher $\mathrm{T}_{\mathrm{dmax}}(\mathrm{c})$ values than $\mathrm{T}_{\mathrm{dmax}}(\mathrm{b})$ values of slight and high crosslinked ALG/dECM composite membranes were observed at $400^{\circ} \mathrm{C}$, which might be contributed to the formation of new mixed corosslinked network microstructures of ALG molecules and dECM molecules. 
When a little amount of ECM was introduced into the ALG/dECM composite membrane without $\mathrm{CaCl}_{2}$, weak ionic association between - $\mathrm{COOH}$ group of ALG molecule and $-\mathrm{NH} 2$ group of $\mathrm{dECM}$ molecule was formed and which is difficult to build up the crosslinking structure[Figure 8(A)]. With an increasing additions of ECM to ALG/dECM composite membranes, ordinary ionic association between -COOH group of ALG molecule and $-\mathrm{NH}_{2}$ group of dECM molecule was employed to build weak ionic crosslinking microstructure[Figure 8(B)]. When a large amount of dECM was introduced into the ALG/dECM composite membrane without $\mathrm{CaCl}_{2}$, strong ionic association between - $\mathrm{COOH}$ group of ALG molecule and $-\mathrm{NH} 2$ group of dECM molecule was employed to build up strong ionic crosslinking microstructure as shown in Fig. $8(\mathrm{C})$.

When a little amount of ECM was introduced into the ALG/dECM composite membrane with $1 \mathrm{wt} \% \mathrm{CaCl}_{2}$, weak ionic association between - $\mathrm{COOH}$ group of ALG molecule and $-\mathrm{NH}_{2}$ group of dECM molecule and weak ionic associations among - $\mathrm{COOH}$ group of ALG molecule, $\mathrm{Ca}^{2+}$, and - $\mathrm{COOH}$ group of ALG molecule could be found. However, the remarkable crosslinked microstructure is still difficult to be observed as shown in Fig. 9(A). $T_{d \max }(\mathrm{C})$ value of ALG/dECM could not be found in Fig. 6(c). With an increasing additions of $\mathrm{CaCl}_{2}$ to $\mathrm{ALG} / \mathrm{dECM}$ composite membranes, weak ionic association between - $\mathrm{COOH}$ group of $A L G$ molecule and $-\mathrm{NH} 2$ group of dECM molecule and weak ionic associations among - $\mathrm{COOH}$ group of ALG molecule, $\mathrm{Ca} 2+$, and $-\mathrm{COOH}$ group of ALG molecule were employed to build weak ionic crosslinking microstructure as shown in Fig. 9(B) and Fig. 7(A). The overlapped $T_{d m a x}(b, c)$ values of ALG/dECM could be observed at $390^{\circ} \mathrm{C}$ in Fig. 7(A). With an increasing addition of dECM to ALG/dECM composite membranes with $5 \mathrm{wt} \% \mathrm{CaCl}_{2}$, some strong ionic associations were employed to build strong mixed ionic crosslinked microstructure. The remarkable high $\mathrm{T}_{\text {dmax }}(\mathrm{c})$ values of $\mathrm{ALG} / \mathrm{dECM}$ could be observed at $400^{\circ} \mathrm{C}$ in Fig. 7(B) 7(D). That is, when the high concentrations of $\mathrm{CaCl}_{2}(5 \mathrm{wt} \%)$ and dECM molecules were added, some different associations would be enhanced, such as association between $\mathrm{COOH}$ group of $\mathrm{ALG}$ molecule and $-\mathrm{NH} 2$ group of $\mathrm{dECM}$ molecule, associations among $-\mathrm{COOH}$ group of ALG molecule, $-\mathrm{COOH}$ group of dECM molecule, and $\mathrm{Ca} 2+$ ion, associations among $-\mathrm{COOH}$ group of ALG molecule, $-\mathrm{COOH}$ group of ALG molecule, and $\mathrm{Ca} 2+$ ion, and associations among $-\mathrm{COOH}$ group of dECM molecule, $-\mathrm{COOH}$ group of dECM molecule, and $\mathrm{Ca} 2+$ ion as shown in Fig. 9(D).

With an increasing additions of dECM to ALG/dECM composite membranes with $1 \mathrm{wt} \% \mathrm{CaCl}_{2}$, ordinary ionic association between - $\mathrm{COOH}$ group of ALG molecule and - $\mathrm{NH} 2$ group of $\mathrm{dECM}$ molecule was employed to build weak ionic crosslinked microstructure. When a large amount of dECM was introduced into the $\mathrm{ALG} / \mathrm{dECM}$ composite membrane with $1 \mathrm{wt} \% \mathrm{CaCl}_{2}$, some ordinary ionic associations such as ionic association between - $\mathrm{COOH}$ group of $\mathrm{ALG}$ molecule and $-\mathrm{NH} 2$ group of dECM molecule, ionic between among $-\mathrm{COOH}$ group of ALG molecule, $\mathrm{Ca}^{2+}$, and $-\mathrm{COOH}$ group of dECM molecule, and ionic between among $-\mathrm{COOH}$ group of dECM molecule, $\mathrm{Ca}^{2+}$, and $-\mathrm{COOH}$ group of dECM molecule as shown in Fig. $9(\mathrm{C})$. The remarkable high $\mathrm{T}_{\mathrm{dmax}}(\mathrm{C})$ values of $\mathrm{ALG} / \mathrm{dECM}$ could be observed at $400{ }^{\circ} \mathrm{C}$ in Fig. $6(\mathrm{~B})$ $\sim 6(\mathrm{D})$. 
For $A L G / d E C M$ without $\mathrm{CaCl}_{2}$, the weight loss were found to be in the range of $70 \%$ and $80 \%$ that was reduced to the residue weight in the range of 20 and $30 \%$ as shown in Fig. 5. For ALG/dECM with CaCl2, this weight loss were found to be in the range of $55 \%$ and $78 \%$ that was reduced to the residue weight in the range of 28 and $45 \%$ as shown in Figs. 6 and 7. The introduction of $\mathrm{CaCl} 2$ could enhance the crosslinking reaction with $\mathrm{CaCl} 2$ and $A L G / d E C M(A L G / E C M 1 L$ and $A L G / E C M 1 H)$, increased the thermal stability of the composite membranes.

\section{Conclusions}

In this study, a series of new composite membranes with collagen scaffolds were successfully obtained from alginate and porcine skin by using supercritical carbon dioxide fluid technology. The retain extracellular matrix and integrity scaffold-structure was observed. This work provides a simple and timesaving method process for decellularized tissue. The network-scaffold microstructures were observed in new composite membranes with collagen scaffolds. The $T_{d \max }$ of the resulting composite membranes could be up to $300^{\circ} \mathrm{C}$. The composite membranes with high thermal stability were obtained. The resulting composite membranes with scaffold microstructures could be a potential application for gene therapy.

\section{References}

[1] J. Malda, 25th anniversary article: Engineering hydrogels for biofabrication. Adv. Mater. 25, 50115028 (2013).

[2] T. Jiang, J. G. Munguia-Lopez, S. Flores-Torres, J. Grant, S. Vijayakumar, A. D. Leon-Rodriguez \& J. M. Kinsella. Directing the self-assembly of tumor spheroids by bioprinting cellular heterogeneous models within Alginate/Gelatin hydrogels. Sci. Rep. 7, 4575 (2017).

[3] C. Mandrycky, Z.Wang, K. Kim, D.H. Kim, 3D bioprinting for engineering complex tissues. Biotechnil. Adv. 34, 422-423 (2016).

[4] Y.W. Liu, C.C. Huang, Y.Y. Wang, J. Xu, G.D. Wang and X.P. Bai, Biological evaluations of decellularized extracellular matrix collagen microparticles prepared based on plant enzymes and aqueous two-phase method, Regenerative Biomaterials, 8(2021), 1-10

[5] D. J. Liaw, C. C. Huang, W. F. Lee, J. Borbély and E. T. Kang, Synthesis and Characteristics of the Poly(carboxybetaine)s and the Corresponding Cationic Polymers, J. Polym. Sci., Part A: Polym. Chem., 35 (1997), 3527-3536

[6] D. J. Liaw, C. C. Huang, H. C. Sang and E. T. Kang, Intramolecular Hydrophobic Aggregation of Amphiphilic Polysulfobetaine with Various Hydrophobic Groups in Aqueous Solution, Langmuir, 15 (1999), 5204-5211 
[7]D. J. Liaw, T. P. Chen, and C. C. Huang, Self-Assembly Aggregation of Highly Stable Copolynorbornenes with Amphiphilic Architecture via Ring-Opening Metathesis Polymerization, Macromolecules. 38(2005), 3533-3538

[8] G. Zhai, S. C. Toh, W. L. Tan, E. T. Kang, K. G. Neoh, C. C. Huang and D. J. Liaw, Poly(vinylidene fluoride) with Grafted Zwitterionic Polymer Side Chains for Electrolyte-Responsive Microfiltration Membranes, Langmuir, 19(2003), 7030-7037

[9] D. J. Liaw, C. C. Huang and E. T. Kang, Novel Fluorescent Polynorbornenes with Multi-functional Armed Structure by Using Highly Stable Block Macroinitiators via A Combination of Living Ring-Opening Metathesis Polymerization and Atom Transfer Radical Polymerization,Polymer,37(2006), 3057-3064

[10] Z. F. Li, E. T. Kang, K. G. Neoh, K. L. Tan, C. C. Huang and D. J. Liaw, Surface Structures and AdhesiveFree Adhesion Characteristics of Polyaniline Films after Modification by Graft Copolymerization, Macromolecules, 30(1997), 3354-3362

[11] G. Zhai, S. C. Toh, W. L. Tan, E. T. Kang, K. G. Neoh, C. C. Huang and D. J. Liaw, 2003, " Poly(vinylidene fluoride) with Grafted Zwitterionic Polymer Side Chains for Electrolyte-Responsive Microfiltration Membranes ", Langmuir, 19(2003), 7030-7037

[12] O. Smidsrød, G. Skjak-Braek, Alginate as immobilization matrix for cells, Trends Biotechnol, 8(1990):71-78.

[13]Qiang-Song Wang, Xiao-Ning Zhu, Heng-Li Jiang, Gui-Fang Wang, Yuan-Lu Cui,Protective effects of alginate-chitosan microspheres loaded with alkaloids from Coptis chinensis Franch. and Evodia rutaecarpa (Juss.) Benth. (Zuojin Pill) against ethanol-induced acute gastric mucosal injury in rats, Drug Design, Development and Therapy, 9(2015) 6151-6165.

[14] J. Wu, T. Kong, K. W. Yeung, Fabrication and characterization of monodisperse PLGA-alginate coreshell microspheres with monodisperse size and homogeneous shells for controlled drug release, Acta Biomater, 9(2013):7410-7419.

[15] K.Y. Lee, D. J. Mooney, Alginate: Properties and biomedical applications. Prog Polym Sci, 2012;37:106-26.

[16] A.K. Anal, W.F.Stevens, Chitosan-alginate multilayer beads for controlled release of ampicillin. Int J Pharm. 290(2005), 45-54.

[17]C.C. Huang, C. Y. Liu, C.Y. Huang, H.W. Liu , Carbodiimide crosslinked and biodegradation-controllable small intestinal submucosa sheets, Journal Bio-Medical Materials and Engineering, 2014, 24 (6): 19591967.

[18] N.P.Camacho, P.West, P.A. Torzilli, R.Mendelsohn, Biopolymers, 62(2001),1-8 
[19] Vogel K G, Trotter J A. The Effect of Proteoglycans on the Morphology of Collagen Fibrils Formed In Vitro. Collagen and related research, 7(1987),105-114.

[20]A.D.Theocharis, S. S.Skandalis, C.Gialeli, N. K.Karamanos, Extracellular matrix structure, Advanced Drug Delivery Reviews, 97(2016), 4-27

\section{Figures}



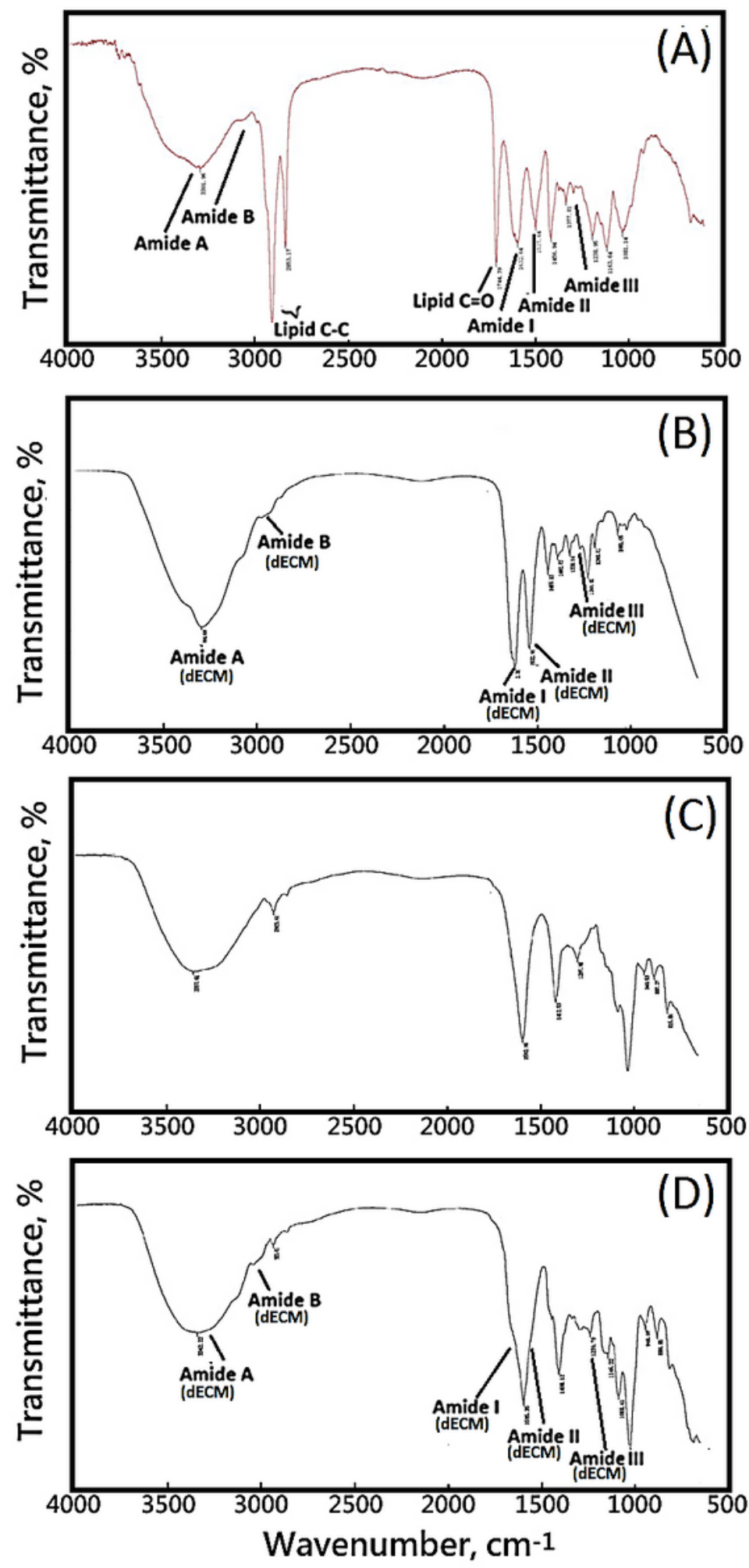

Figure 1

FTIR Spectrum of thesamples: (A)original porcine skin, (B)dECM, (C)ALG/dECMON, and (D)cross-linked ALG/dECM composite membrane(ALG/dECM4H). 

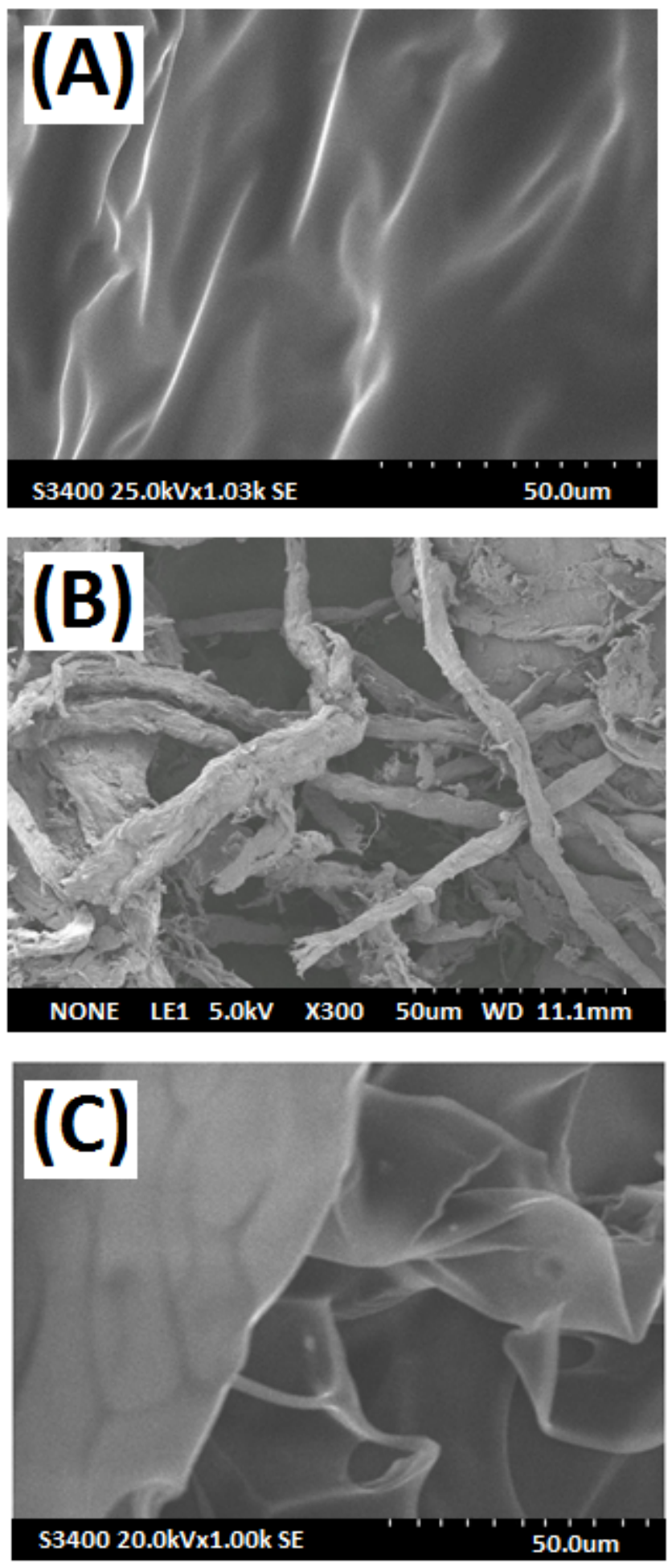

Figure 2

Scanning electron micrographs of the samples (a)original porcine skin, (b)dECM powder, and (c) ALG/dECMON membrane. 

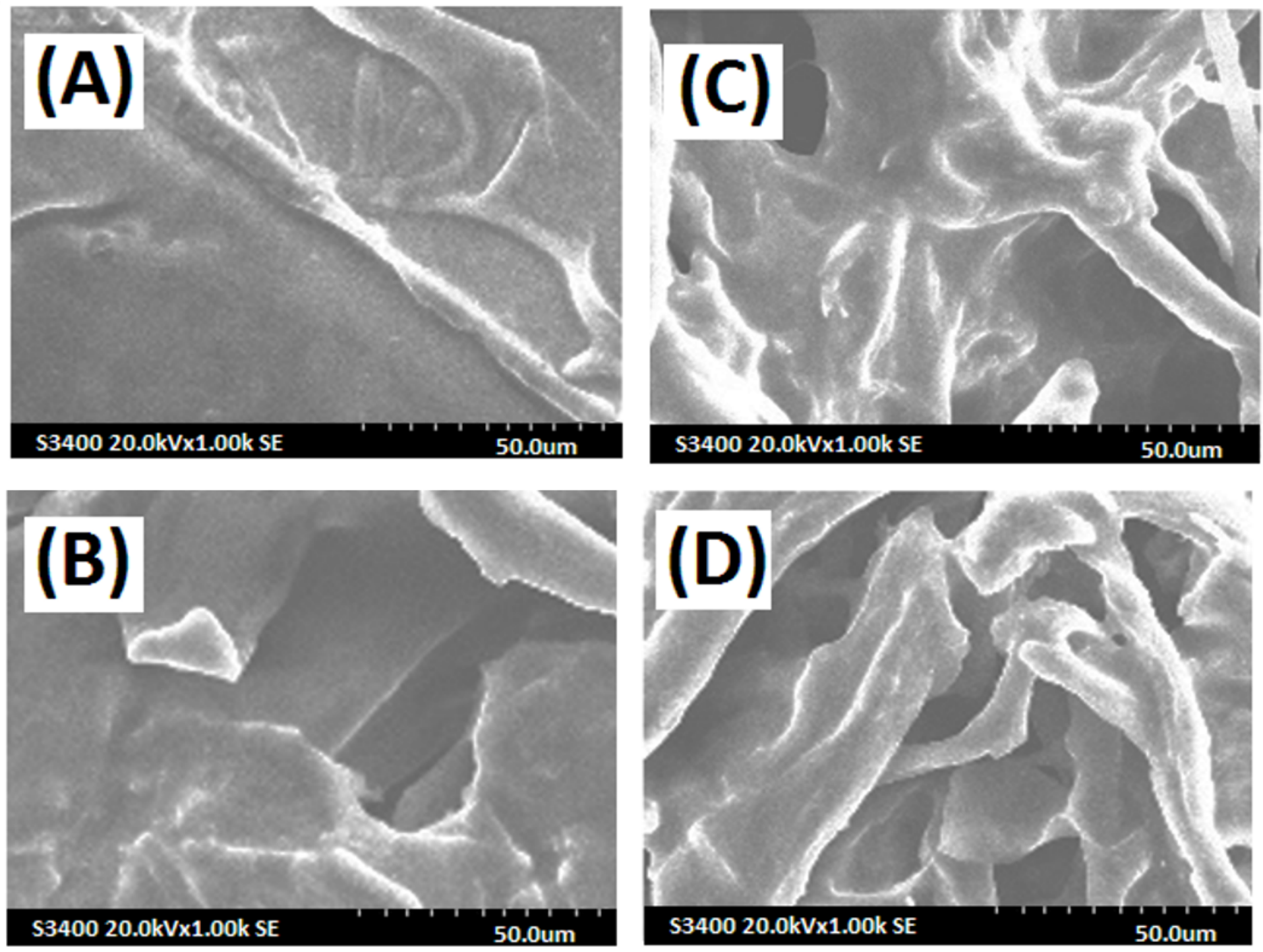

\section{Figure 3}

Scanning electron micrographs of the ALG/dECM composite membrane samples (A) ALG/dECM1H, (B) ALG/dECM2H, (C) ALG/dECM3H AND (D) ALG/dECM4H. 

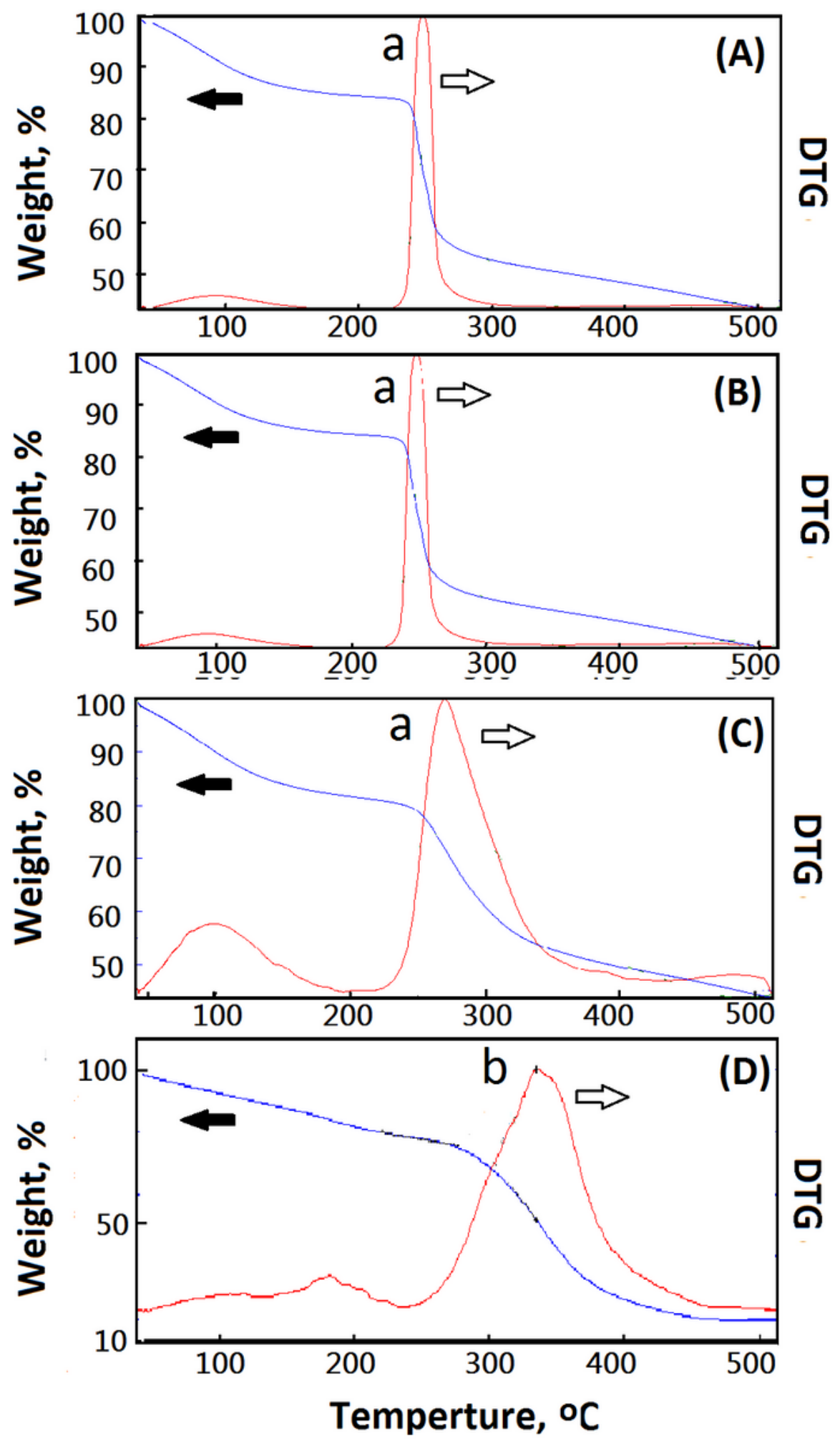

Figure 4

Thermogravimetric analysis of the composite membranes with/without dECM: (A)ALG/dECMON, (B)ALG/dECMOL, (C)ALG/dECMOH, and (D)dECM. 


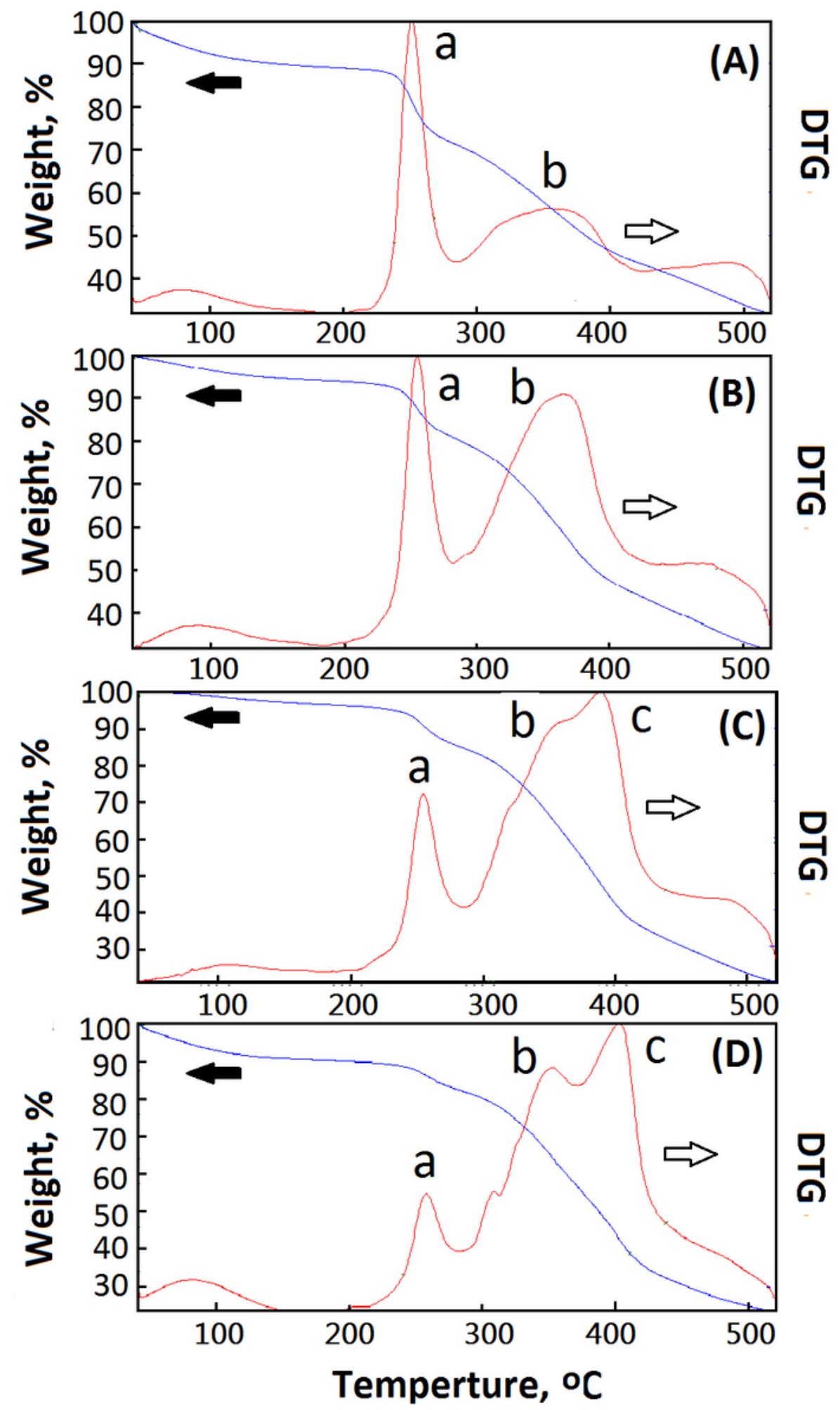

Figure 5

Thermogravimetric analysis of the composite membranes: (A)ALG/dECM1N, (B)ALG/dECM2N, (C)ALG/dECM3N, and (D)ALG/dECM4N. 


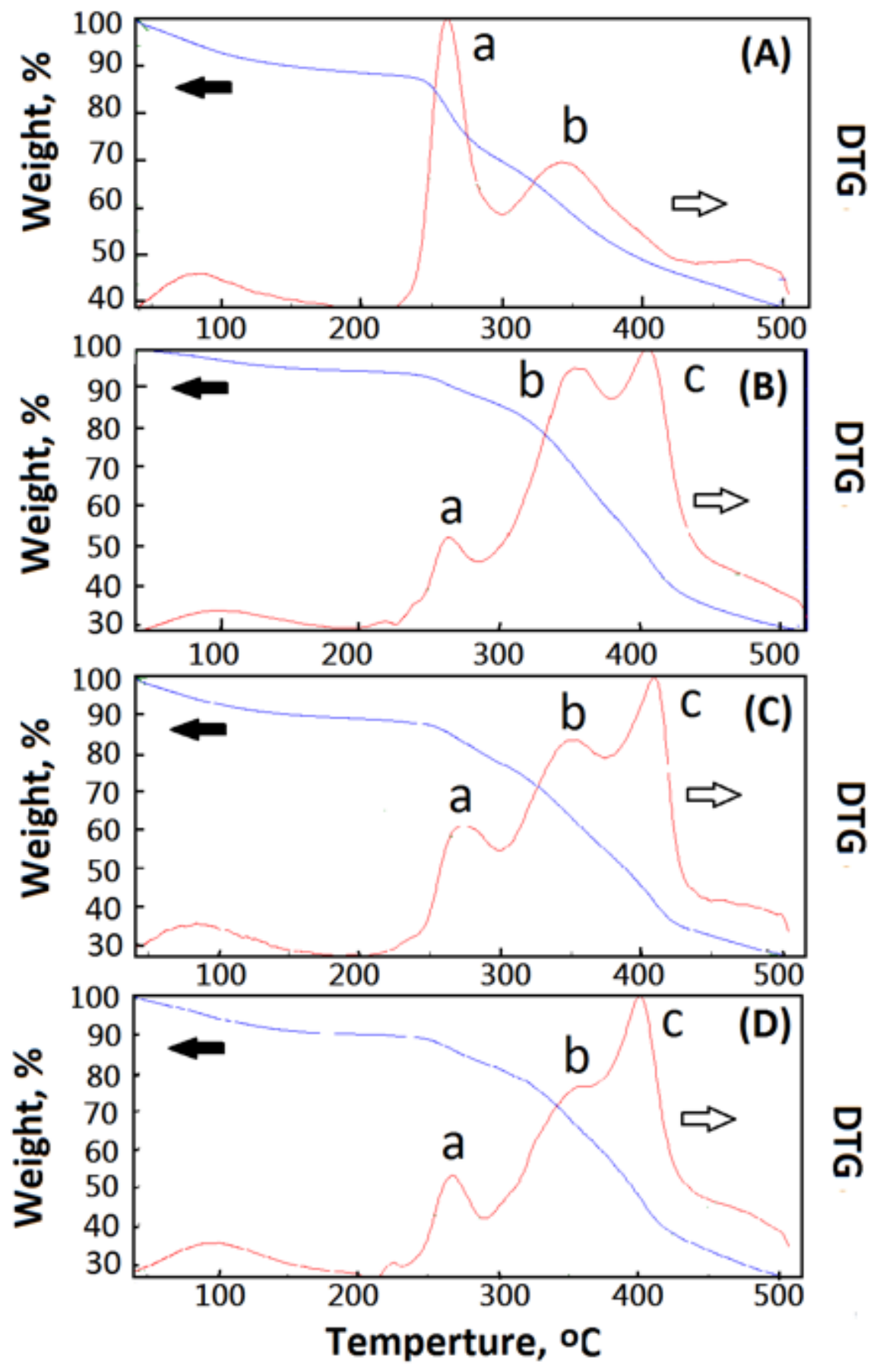

Figure 6

Thermogravimetric analysis of the composite membranes: (A)ALG/dECM1L, (B)ALG/dECM2L, (C)ALG/dECM3L, and (D)ALG/dECM4L. 

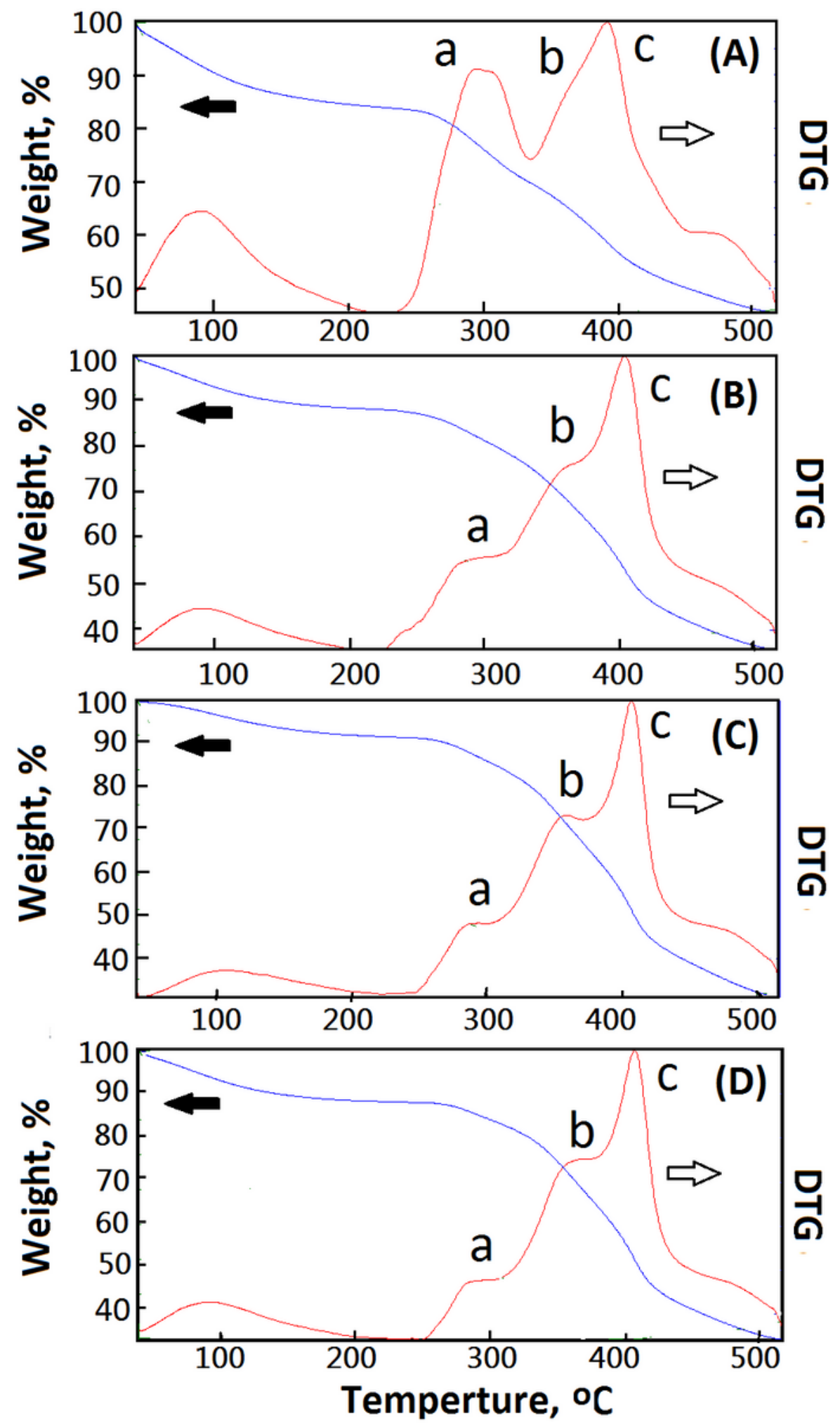

Figure 7

Thermogravimetric analysis of the composite membranes: (A)ALG/dECM1H, (B)ALG/dECM2H, (C)ALG/dECM3H, and (D)ALG/dECM4H. 


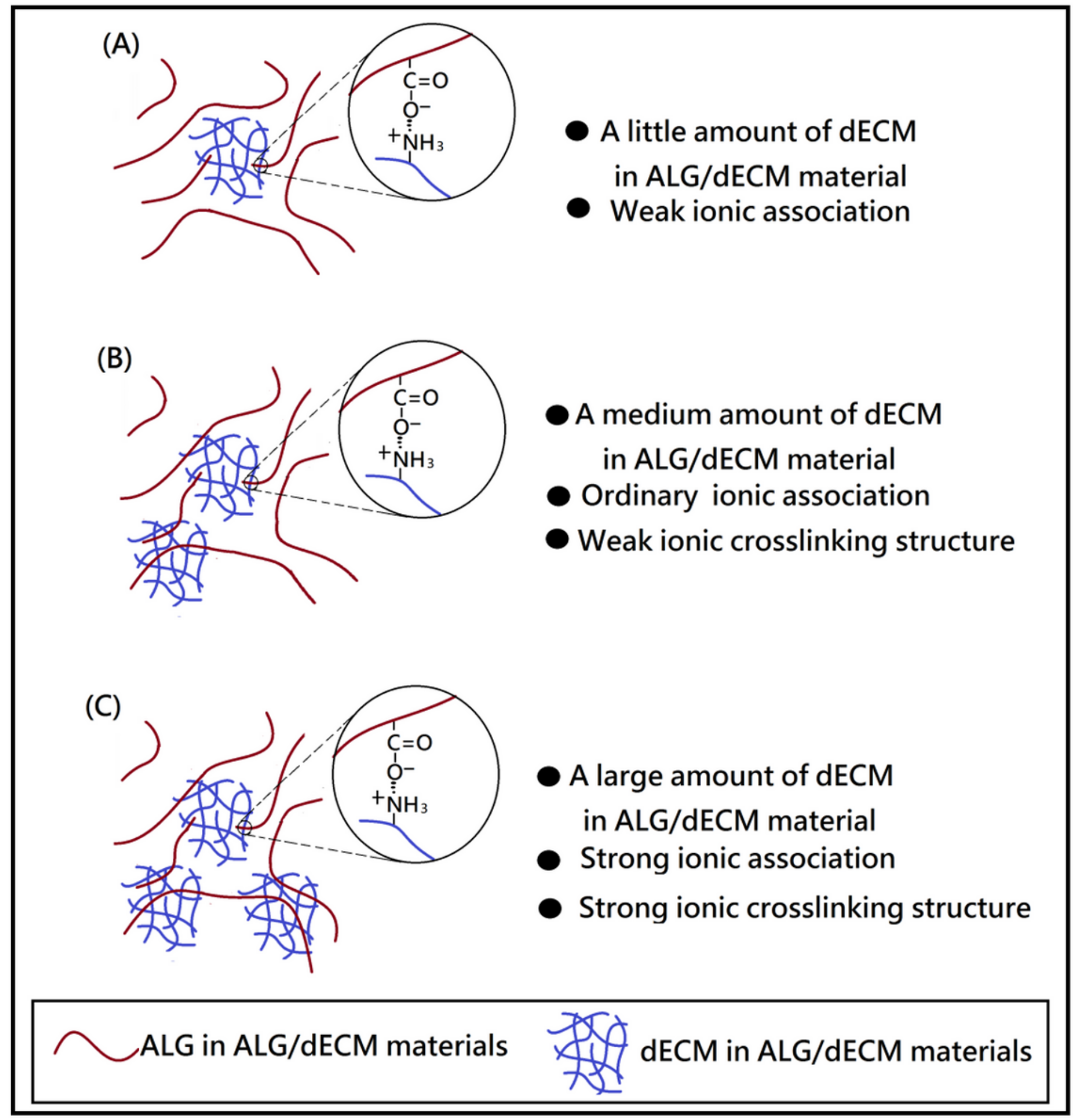

Figure 8

Proposed model of ionic associations between ALG molecule and AECM molecule within designed composite membranes without $\mathrm{CaCl} 2$. 


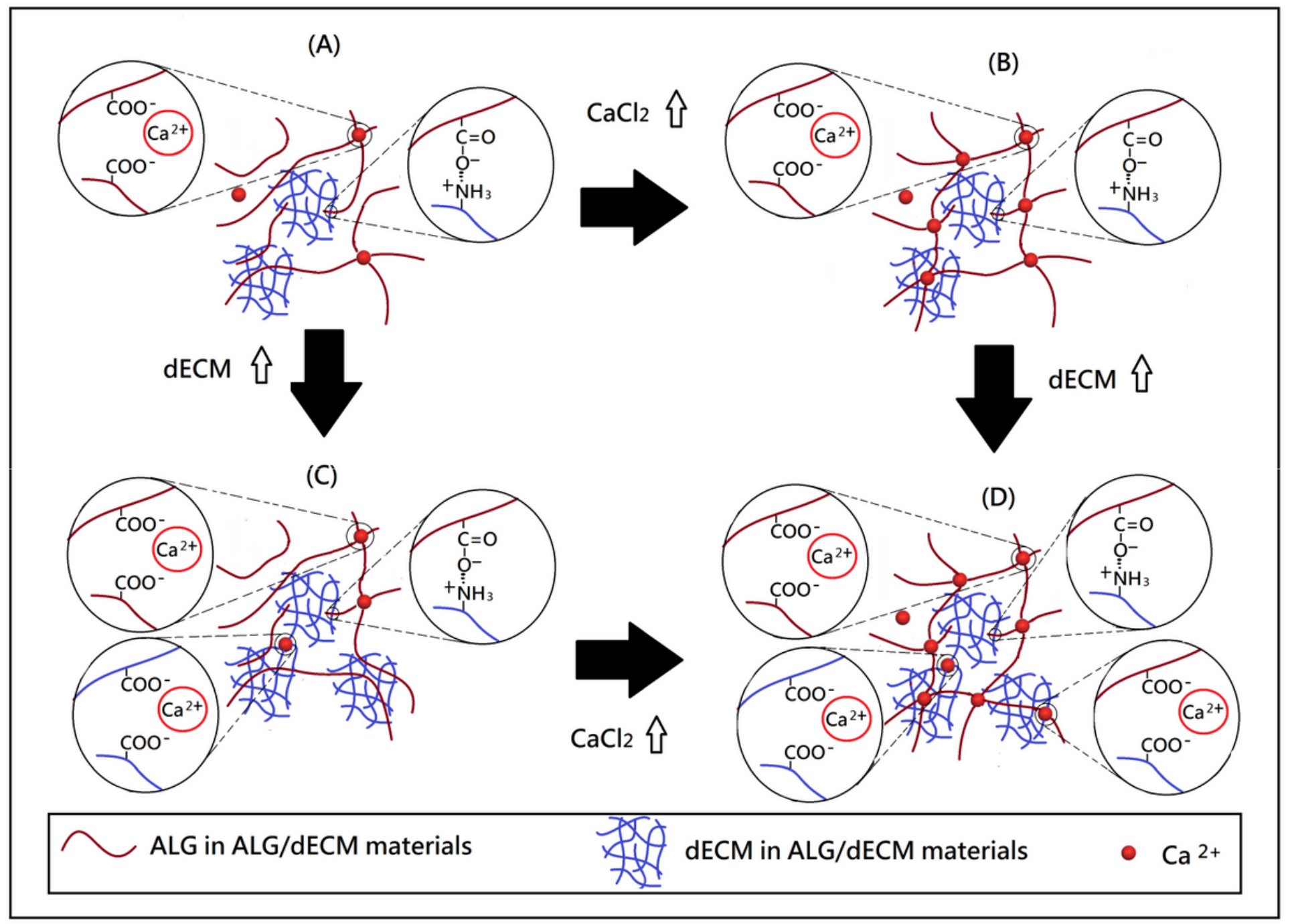

Figure 9

Proposed model of ionic associations among Ca2+, ALG molecule, and dECM molecule within designed composite membranes. 